\title{
Rett mutations attenuate phase separation of $\mathrm{MeCP} 2$
}

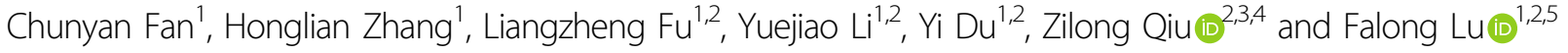

\section{Dear Editor,}

Methyl-CpG-binding protein 2 (MeCP2) is a ubiquitously expressed nuclear protein originally identified as a methylated DNA binding protein, which is particularly abundant in mature neurons ${ }^{1,2}$. Deficiency or excess of MeCP2 causes severe neurological problems. Mutations in $\mathrm{MeCP} 2$ account for $95 \%$ of the dominant X-linked neurological disorder Rett syndrome ${ }^{3}$. MeCP2 have two key functional domains: the methyl-DNA binding domain (MBD) and the transcriptional repressor domain (TRD). Almost all of missense Rett mutations are clustered in these two domains, such as R133C, F155S, T158M in $\mathrm{MBD}$, and $\mathrm{R} 306 \mathrm{H}$ in $\mathrm{TRD}^{4,5}$. The mechanism of the mutations leading to Rett syndrome is still not well understood. Here, we reveal that MeCP2 can drive the liquid-liquid phase separation (LLPS) in complex with DNA. Interestingly, this ability is compromised in the presence of mutations found in Rett syndrome patients, suggesting a potential common mechanism by disrupting LLPS of MeCP2 droplets underlying Rett syndrome.

MeCP2 forms sharp condensed foci which highly overlap with DNA dense compartments in neuronal nuclei ${ }^{6,7}$. Recently, LLPS has been recognized as an important mechanism to condensate molecules to form membraneless compartments within a cell ${ }^{8}$. As both the MBD and TRD of MeCP2 bind to DNA ${ }^{9}$, we hypothesized that the sharp puncta of MeCP2 in the nuclei were phase separated liquid droplets mediated by multivalent interactions between MeCP2 and DNA. To test this hypothesis, we purified full-length recombinant His-MBPMeCP2 (mouse MeCP2-e2 if not specified) and released

\footnotetext{
Correspondence: Falong Lu (fllu@genetics.ac.cn)

${ }^{1}$ State Key Laboratory of Molecular Developmental Biology, Institute of

Genetics and Developmental Biology, Chinese Academy of Sciences, 100101 Beijing, China

'University of Chinese Academy of Sciences, 100049 Beijing, China

Full list of author information is available at the end of the article

These authors contributed equally: Chunyan Fan, Honglian Zhang
}

the His-MBP tag by cutting with TEV protease (Supplementary Fig. S1). CpG methylated DNA was prepared by methylating a $1.1 \mathrm{~kb}$ DNA fragment with the M.SssI methyltransferase and verified by digesting with a methylation sensitive endonuclease, BsiWI (Supplementary Fig. S2). Phase separation was assayed using different concentration of a $1.1 \mathrm{~kb}$ unmodified or methylated DNA with $\mathrm{MeCP} 2$ in a buffer containing $150 \mathrm{mM} \mathrm{NaCl}$ and a low concentration of DAPI for visualizing DNA. Puncta formation was observed both in the presence of DNA or methylated DNA (Fig. 1a). In contrast, MeCP2 protein alone and DNA alone could not form the puncta (Supplementary Fig. S3a, b). In addition, His-MBP in mixture with mEGFP protein with or without DNA could not form puncta either (Supplementary Fig. S3c), confirming that MeCP2 together with DNA or methylated DNA drives the puncta formation.

Next, we monitored the property of the puncta in more details. The MeCP2/DNA droplets can fuse together upon contact (Fig. 1b). Moreover, we observed quick recovery of EGFP fluorescence after photo-bleaching of the middle of MeCP2-mEGFP/DNA droplets (Fig. 1c). These evidences support that the puncta formed by MeCP2 and DNA are LLPS droplets. To compare the ability of DNA and methylated DNA in promoting the phase separation of MeCP2, we calculated 'Occupancy Rate' of the droplets formed by MeCP2 with methylated DNA over unmethylated DNA. We found that about $66 \%$ values were positive (stronger) and $25 \%$ values were negative (weaker), indicating that $\mathrm{CpG}$ methylation could moderately promote LLPS of MeCP2 in complex with DNA (Fig. 1d). Taken together, these data reveal that MeCP2 can undergo LLPS with DNA which can be moderately enhanced by methylation at CpG sites.

Rett mutations have been found both within and outside the MBD domain ${ }^{4}$. Therefore, the loss of the binding to methylated $\mathrm{CpG}$ is not the sole mechanism of MeCP2 


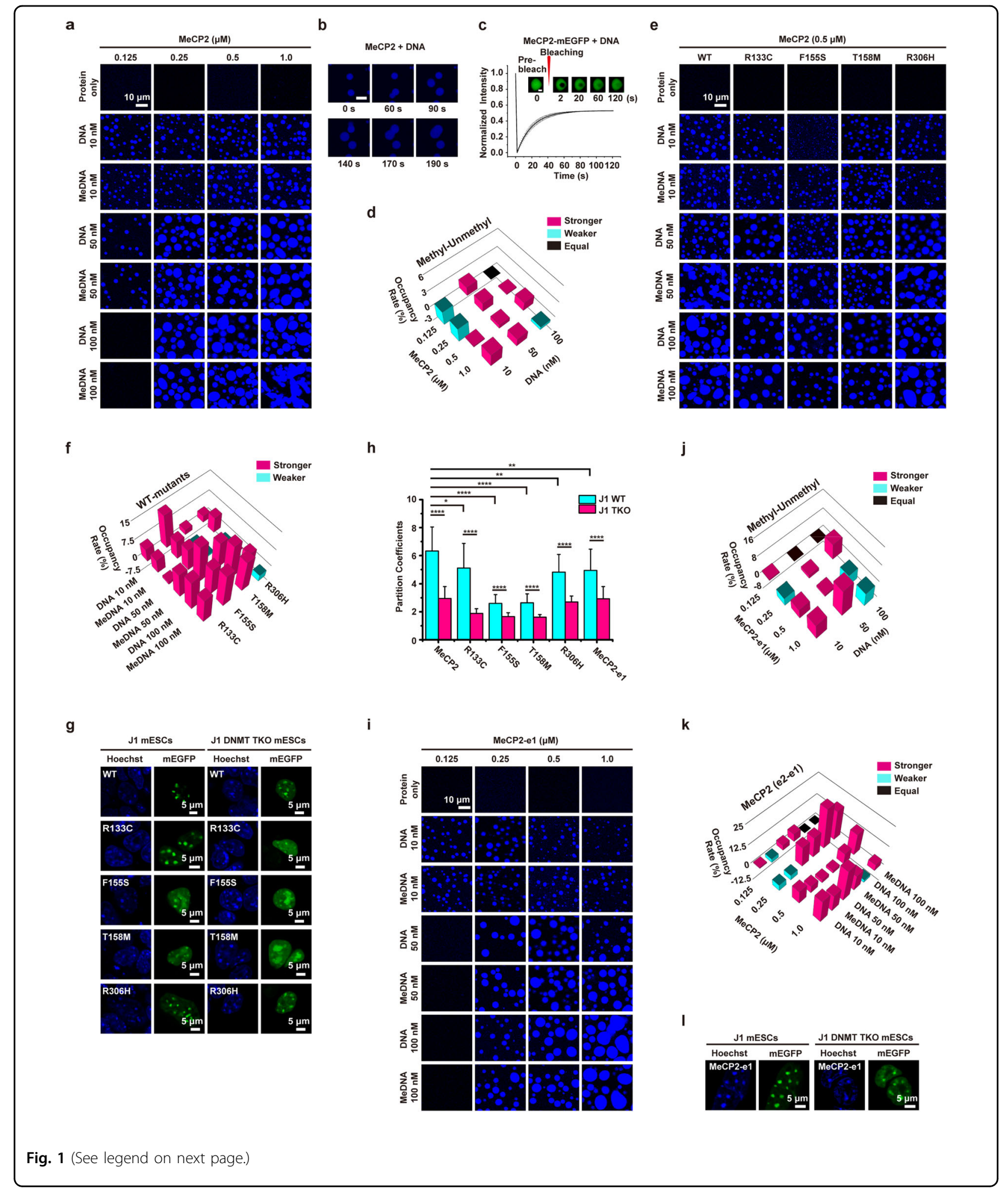

mutations in causing Rett syndrome. We asked whether the phase separation impairment can be a common result of Rett mutations. We examined the effect of three missense mutations in the MBD domain (R133C, F155S and
T158M) and one in the TRD domain (R306H) (Supplementary Fig. S4). MeCP2 proteins with the above Rett mutations were mixed with DNA or methylated DNA to explore whether Rett mutations affect the phase 
(see figure on previous page)

Fig. 1 Rett mutants attenuate phase separation of MeCP2. a Phase diagram of MeCP2 with unmethylated DNA or methylated DNA (MeDNA) in $150 \mathrm{mM} \mathrm{NaCl}$. DAPI was used for DNA visualization. Scale bar, $10 \mu \mathrm{m}$. b Fusion of droplets formed by MeCP2 protein (1.0 $\mu \mathrm{M})$ with DNA (100 nM) in $150 \mathrm{mM} \mathrm{NaCl}$. Scale bar, $5 \mu \mathrm{m}$. c FRAP analysis of droplets formed by MeCP2-mEGFP protein $(1.0 \mu \mathrm{M})$ with DNA (100 nM) after incubating $5 \mathrm{~min}$ on 384-well microplates. Scale bar, $2 \mu \mathrm{m}$. All data are presented as mean \pm SD $(n=3)$. $\mathbf{d}$ Comparison of phase diagrams of MeCP2 with methylated DNA (MeDNA) and unmethylated DNA in Fig. 1a. Pink, cyan, and black squares indicate stronger, weaker and unchanged LLPS. e Phase diagram of MeCP2 wild-type and Rett mutant proteins $(0.5 \mu \mathrm{M})$ with DNA or MeDNA in $150 \mathrm{mM} \mathrm{NaCl}$. DAPI was used for DNA visualization. Scale bar, $10 \mu \mathrm{m}$. $\mathbf{f}$ Comparison of phase diagrams of MeCP2 wild-type and mutant proteins $(0.5 \mu \mathrm{M})$ with DNA or MeDNA in (e). $\mathbf{g}$ Subcellular localization of MeCP2 and Rett mutants tagged with mEGFP overexpressed in $\mathrm{J1}$ wild-type and DNMT TKO mESCs. Hoechst was used to stain DNA. Scale bar, $5 \mu m$. h Partitioning quantification of MeCP2-mEGFP proteins. ${ }^{* * *} p<0.0001,{ }^{* * *} p<0.001,{ }^{* *} p<0.01,{ }^{*} p<0.1$. i Phase diagram of MeCP2-e1 with DNA or MeDNA in $150 \mathrm{mM} \mathrm{NaCl}$. DAPI was used for DNA visualization. Scale bar, $10 \mu \mathrm{m}$. j Comparison of phase diagrams of MeCP2-e1 with MeDNA and DNA in (i). $\mathbf{k}$ Comparison of phase diagrams of MeCP2-e2 and MeCP2-e1 with DNA or MeDNA in (a, i). I Subcellular localization of MeCP2-e1-mEGFP overexpressed in $\mathrm{J1}$ wild-type and DNMT TKO mESCs. Scale bar, $5 \mu \mathrm{m}$.

separation of MeCP2. We found that the phase separation of these mutants was weaker than that of the wild-type protein under the same conditions (Fig. 1e, f, Supplementary Fig. S3d), indicating that the Rett mutations attenuate phase separation of MeCP2 in vitro.

In order to study whether Rett mutations affect the condensation of MeCP2 in vivo, we transfected wild-type MeCP2 and Rett mutants tagged with mEGFP to J1 mESCs (mouse embryonic stem cells). Western blot with MeCP2 antibody showed that the exogenous MeCP2-mEGFP level was slightly higher than the endogenous MeCP2 level in J1 mESCs, but lower than that in adult mouse brain sample (Supplementary Fig. S5), indicating that the MeCP2-mEGFP level was within the range of physiological level. MeCP2 formed sharp puncta preferentially associated with Hoechst-dense foci in vivo (Fig. 1g), which was consistent with previous studies ${ }^{6,7}$. F155S and T158M mutants were partially excluded from Hoechst-dense region and mis-localized to nucleoli similar to previous observation of a MBD domain deletion mutant ${ }^{7}$. The $\mathrm{R} 133 \mathrm{C}$ and $\mathrm{R} 306 \mathrm{H}$ mutants remained mainly in Hoechst-dense region (Fig. 1g). FRAP analysis of the puncta indicated that the mobility of the MeCP2 within puncta was enhanced in the presence of Rett mutations (Supplementary Fig. S6, Table S1). Quantifying the condensation of MeCP2 and the mutants using partition coefficient' approach ${ }^{10}$, the puncta of the Rett mutants showed obvious reduced condensation compared to WT protein (Fig. 1h), indicating that Rett mutations impaired the condensation of MeCP2 in vivo.

To test whether DNA methylation further affect the puncta of MeCP2 in vivo, we took advantage of J1 DNMT TKO (Dnmt $1^{-/}$Dnmt3a $a^{-/}$Dnmt $\left.3 b^{-/-}\right)$mESCs in which all the major DNA methyltransferases (DNMT1, DNMT3A, and DNMT3B) were depleted, while the chromatin structure was minimally affected ${ }^{11,12}$. MeCP2 wild-type, $\mathrm{R} 133 \mathrm{C}$ and $\mathrm{R} 306 \mathrm{H}$ still formed puncta at Hoechst-dense foci in J1 DNMT TKO mESCs, while F155S and T158M mutants were mostly excluded from
Hoechst-dense region (Fig. 1g). FRAP analysis showed the mobility of MeCP2 was enhanced in DNMT TKO cells compared to that in WT cells (Supplementary Fig. S7, Table S1). Compared to puncta seen in wild-type mESCs, the condensation of MeCP2 and Rett mutants expressed in DNMT TKO mESCs were weakened (Fig. 1g, h). These data are consistent with the in vitro results that methylated DNA likely further enhances LLPS of MeCP2 compared to unmodified DNA. We noted that DNA methylation showed larger impact on the condensation of $\mathrm{MeCP} 2$ in vivo than in vitro (Fig. 1a, d, g, h), suggesting additional regulation of $\mathrm{MeCP} 2$ condensation in vivo. Together, these data show that DNA methylation can enhance the condensation of MeCP2 in vivo.

The MeCP2 gene gives rise to two different isoforms with different $\mathrm{N}$-terminals due to alternative inclusion of exon 2 in e2 isoform (MeCP2-e1 and MeCP2-e2) ${ }^{6}$ (Supplementary Fig. S4). We asked if MeCP2-e1 was with different ability in driving LLPS as MeCP2-e2. We found that DNA binding could drive MeCP2-e1 to form LLPS, while CpG methylation could slightly enhance the LLPS (Fig. 1i, j, Supplementary Fig. S3e). Interestingly, the phase separation of MeCP2-e1 was moderately weaker than that of MeCP2-e2 (Fig. 1k). MeCP2-e1 tagged with mEGFP formed puncta in mESCs similarly to MeCP2-e2, but with weaker condensation and higher mobility (Fig. 1g, h, l, Supplementary Fig. S8, Table S1). The different ability of the two MeCP2 isoforms in driving LLPS may be related to the difference of their DNA binding affinity ${ }^{13}$, suggesting functional divergence of the two isoforms which warrants future study.

In this study, we reveal that MeCP2 together with DNA can drive LLPS, which can be moderately enhanced by the presence of $\mathrm{CpG}$ methylation. Interestingly, the two isoforms show different capability in driving LLPS. More interestingly, this ability of MeCP2 in driving LLPS is commonly compromised in the presence of mutations found in Rett syndrome patients both inside and outside MBD domain. A recent report published during revision 
of this study also revealed the impairment of MeCP2 phase separation by Rett mutations ${ }^{14}$. These findings suggest a potential role of $\mathrm{MeCP} 2$ in driving the formation of chromatin compartmentation necessary for normal neuronal function, providing a new angle to understand the mechanism underlying Rett syndrome.

\section{Acknowledgements}

We thank Dr. Zhiheng Xu for anti-MeCP2 antibody, Ms. Ruitian Song for technical assistance, and the Center for Biological Imaging, Institute of Biophysics for confocal microscopy assistance. This work was supported by the National Key Research and Development Program of China (2018YFA0107001), the National Natural Science Foundation of China (31900419, 81891001).

\section{Author details}

'State Key Laboratory of Molecular Developmental Biology, Institute of Genetics and Developmental Biology, Chinese Academy of Sciences, 100101 Beijing, China. ${ }^{2}$ University of Chinese Academy of Sciences, 100049 Beijing, China. ${ }^{3}$ Chinese Academy of Sciences Center for Excellence in Brain Science and Intelligence Technology, Chinese Academy of Sciences, 200031 Shanghai, China. ${ }^{4}$ State Key Laboratory of Neuroscience, Institute of Neuroscience, Chinese Academy of Sciences, 200031 Shanghai, China. ${ }^{5}$ The Innovative Academy of Seed Design, Chinese Academy of Sciences, 100101 Beijing, China

\section{Author contributions}

F.L., Z.Q., and H.Z. conceived the project and designed the experiments; C.F. performed most of the experiments with H.Z., L.F., Y.L., and Y.D.; H.Z. initially discovered the LLPS of MeCP2; C.F., H.Z., and F.L. wrote the manuscript.

\section{Conflict of interest}

The authors declare that they have no conflict of interest.

\section{Publisher's note}

Springer Nature remains neutral with regard to jurisdictional claims in published maps and institutional affiliations.

Supplementary Information accompanies the paper at (https://doi.org/ 10.1038/s41421-020-0172-0).
Received: 29 December 2019 Accepted: 27 April 2020

Published online: 16 June 2020

\section{References}

1. Lewis, J. D. et al. Purification, sequence, and cellular localization of a novel chromosomal protein that binds to methylated DNA. Cell 69, 905-914 (1992).

2. Kishi, N. \& Macklis, J. D. MECP2 is progressively expressed in post-migratory neurons and is involved in neuronal maturation rather than cell fate decisions. Mol. Cell Neurosci. 27, 306-321 (2004).

3. Weaving, L. S., Ellaway, C. J., Gecz, J. \& Christodoulou, J. Rett syndrome: clinical review and genetic update. J. Med. Genet. 42, 1-7 (2005).

4. Lavery, L. A. \& Zoghbi, H. Y. The distinct methylation landscape of maturing neurons and its role in Rett syndrome pathogenesis. Curr. Opin. Neurobiol. 59, 180-188 (2019).

5. Amir, R. E. et al. Rett syndrome is caused by mutations in X-linked MECP2, encoding methyl-CpG-binding protein 2. Nat. Genet. 23, 185-188 (1999).

6. Kriaucionis, S. \& Bird, A. The major form of MeCP2 has a novel N-terminus generated by alternative splicing. Nucleic Acids Res. 32, 1818-1823 (2004).

7. Kumar, A. et al. Analysis of protein domains and Rett syndrome mutations indicate that multiple regions influence chromatin-binding dynamics of the chromatin-associated protein MECP2 in vivo. J. Cell Sci. 121, 1128-1137 (2008).

8. Alberti, S., Gladfelter, A. \& Mittag, T. Considerations and challenges in studying liquid-liquid phase separation and biomolecular condensates. Cell 176, 419-434 (2019).

9. Hansen, J. C., Ghosh, R. P. \& Woodcock, C. L. Binding of the Rett syndrome protein, MeCP2, to methylated and unmethylated DNA and chromatin. IUBMB Life 62, 732-738 (2010).

10. Pak, C. W. et al. Sequence determinants of intracellular phase separation by complex coacervation of a disordered protein. Mol. Cell 63, 72-85 (2016).

11. Ito, S. et al. Role of Tet proteins in $5 \mathrm{mC}$ to $5 \mathrm{hmC}$ conversion, ES-cell selfrenewal and inner cell mass specification. Nature 466, 1129-1133 (2010).

12. Tsumura, A. et al. Maintenance of self-renewal ability of mouse embryonic stem cells in the absence of DNA methyltransferases Dnmt1, Dnmt3a and Dnmt3b. Genes Cells 11, 805-814 (2006).

13. Martinez de Paz, A. et al. MeCP2-E1 isoform is a dynamically expressed, weakly DNA-bound protein with different protein and DNA interactions compared to MeCP2-E2. Epigenet. Chromatin 12, 63 (2019).

14. Wang, L. et al. Rett syndrome-causing mutations compromise MeCP2mediated liquid-liquid phase separation of chromatin. Cell Res. https://doi.org/ 10.1038/s41422-020-0288-7 (2020). 